\title{
An Experiment of Nature: Brain Anatomy Parallels Cognition and Behavior in Williams Syndrome
}

\author{
Allan L. Reiss, ${ }^{1}$ Mark A. Eckert, ${ }^{1}$ Fredric E. Rose,${ }^{2}$ Asya Karchemskiy, ${ }^{1}$ Shelli Kesler, ${ }^{1}$ Melody Chang, ${ }^{1}$ \\ Margaret F. Reynolds, ${ }^{1}$ Hower Kwon, ${ }^{1}$ and Al Galaburda ${ }^{3}$ \\ ${ }^{1}$ Department of Psychiatry and Behavioral Sciences, Stanford University School of Medicine, Stanford, California 94305, ${ }^{2}$ Laboratory for Cognitive \\ Neuroscience, Salk Institute, La Jolla, California 92037 and ${ }^{3}$ Harvard Medical School, Department of Neurology, Boston, Massachusetts 02215
}

Williams syndrome (WS) is a neurogenetic-neurodevelopmental disorder characterized by a highly variable and enigmatic profile of cognitive and behavioral features. Relative to overall intellect, affected individuals demonstrate disproportionately severe visual-spatial deficits and enhanced emotionality and face processing. In this study, high-resolution magnetic resonance imaging data were collected from 43 individuals with WS and 40 age- and gender-matched healthy controls. Given the distinct cognitive-behavioral dissociations associated with this disorder, we hypothesized that neuroanatomical integrity in WS would be diminished most in regions comprising the visual-spatial system and most "preserved" or even augmented in regions involved in emotion and face processing. Both volumetric analysis and voxel-based morphometry were used to provide convergent approaches for detecting the hypothesized WS neuroanatomical profile. After adjusting for overall brain volume, participants with WS showed reduced thalamic and occipital lobe gray matter volumes and reduced gray matter density in subcortical and cortical regions comprising the human visual-spatial system compared with controls. The WS group also showed disproportionate increases in volume and gray matter density in several areas known to participate in emotion and face processing, including the amygdala, orbital and medial prefrontal cortices, anterior cingulate, insular cortex, and superior temporal gyrus. These findings point to specific neuroanatomical correlates for the unique topography of cognitive and behavioral features associated with this disorder.

Key words: emotion; neuron; imaging; morphometry; visual; Williams syndrome; neuroanatomy

\section{Introduction}

The enigmatic and compelling nature of the neurocognitive phenotype associated with Williams syndrome (WS) has brought this neurogenetic condition to the forefront of interest in the neuroscience community. The fact that WS has a specific and well-defined genetic etiology (interstitial deletion on chromosome 7) provides a rare opportunity for elucidating linkages among genetic, neurobiological, and neurocognitive levels of investigation (Bellugi et al., 1999).

Numerous studies have provided overwhelming evidence that, in relation to mild to moderate intellectual deficits, individuals with WS demonstrate severe impairment in their ability to process visual-spatial stimuli. Dorsal stream dysfunction may account for the poor visual-spatial performance (Atkinson et al., 1997, 2001; Paul et al., 2002). This hypothesis also is supported by a relative strength in face processing (Bellugi et al., 2000), a task that engages the ventral visual stream.

Received Nov. 28, 2003; revised March 31, 2004; accepted April 8, 2004.

The research presented in this manuscript was supported by National Institutes of Health Grants K02 MH01142, R01 HD31715, and P01 HD33113. We extend our particular appreciation to the members of the Brain, Behavior, and Genetics Group who provided invaluable support for this study, including Drs. Ursula Bellugi, Julie Korenberg, Debra Mills, and Mark Appelbaum.

Correspondence should be addressed to Allan Reiss, Stanford University School of Medicine, Department of Psychiatry and Behavioral Sciences, 401 Quarry Road, Stanford, CA 94305. E-mail: reiss@stanford.edu. DOI:10.1523/JNEUROSCI.5272-03.2004

Copyright $\odot 2004$ Society for Neuroscience $\quad$ 0270-6474/04/245009-07\$15.00/0
Although not as abundant as studies demonstrating atypical visual-spatial function, recent investigations have begun to provide empirical support for a long-held "clinical" observation of heightened and aberrant emotional function in WS, particularly within the contexts of socialization and communication. For example, persons with WS are at increased risk for significant, longterm problems with excessive anxiety and over-arousal compared with intelligence quotient (IQ)-matched controls (Davies et al., 1998; Dykens, 2003). Individuals with WS also demonstrate excessive linguistic affect during conversations and when giving narratives (Jones et al., 2000; Reilly et al., 2004) and heightened emotional reactions to music and certain classes of noise (Gosch and Pankau, 1994; Einfeld et al., 1997). In a preliminary functional magnetic resonance imaging study from our group (Levitin et al., 2003), we showed that, compared with controls, individuals with WS had significantly increased (right) amygdala activation during music processing compared with controls, thus providing a putative neural correlate of increased emotional "expressiveness" observed within the contexts of both music (Hopyan et al., 2001) and language (Losh et al., 2000) in affected individuals.

To date, studies of brain structure and function have hinted only at the neural correlates of the extraordinary profile of neurocognitive function in WS (Wang et al., 1992a,b; Reiss et al., 2000; Schmitt et al., 2001a,b,c, 2002). In the study presented here, neuroimaging data from 43 individuals with WS and 40 healthy 
controls are examined, focusing on brain regions known to contribute to either visual or emotion-face processing in humans. Given the distinct cognitive dissociations associated with this disorder, we hypothesized that neuroanatomical integrity in WS would be most diminished in regions comprising the visual-spatial system. We secondarily predicted that the anatomy of regions involved in emotion and face processing would be most "preserved" or perhaps even augmented in relation to healthy controls. We used complementary methods for image processing, volumetric analysis and voxel-based morphometry (VBM) (Good et al., 2001), to provide convergent approaches for detecting the hypothesized neuroanatomical profile associated with this disorder.

\section{Materials and Methods}

Participants. All WS participants were recruited and evaluated at the Salk Institute as part of a larger program project examining the associations among behavior, neurophysiology, neuroanatomy, and molecular genetics. Participants were excluded from the study if they had a history of concurrent medical conditions not typically associated with WS, particularly those with confounding medical or neurological consequences such as epilepsy. The diagnosis of WS was genetically confirmed in all participants using the fluorescent in situ hybridization test for a deletion of one copy of the elastin gene on chromosome 7.

Healthy control participants were recruited at both the Salk Institute and Stanford University. These participants had no history of major psychiatric, neurological, or cognitive impairment. The institutional review boards of both institutions approved the procedures. All participants and, if appropriate, their parents or guardians provided informed written consent for the study. Preliminary data from a total of $14 \mathrm{WS}$ and 14 control participants have been reported previously (Reiss et al., 2000).

MRI protocol. MR images of each participant's brain were acquired with a GE-Signa 1.5 T scanner (General Electric, Milwaukee, WI) located at one of three sites: University of California, San Diego Medical Center Magnetic Resonance Imaging Institute $(n=59)$, Scripps Clinic, San Diego $(n=53)$, or Stanford University $(n=5)$. In all cases, sagittal brain images were acquired with the same three-dimensional (3D) volumetric radio frequency spoiled gradient echo (spoiled gradient-recalled acquisition in a steady state) pulse sequence using the following scan parameters: repetition time, $24 \mathrm{msec}$; echo time, $5 \mathrm{msec}$; flip angle, $45^{\circ}$; number of excitations, 2 ; matrix size, $256 \times 192$; field of view, $24 \mathrm{~cm}$; slice thickness, $1.2 \mathrm{~mm}$; 124 contiguous slices. Scans were analyzed at the Stanford Psychiatry Neuroimaging Laboratory by raters blinded to participant group membership. Previous work from our laboratory has demonstrated the compatibility of imaging data acquired from multiple sites when using the same magnetic field strength, pulse sequence, and image processing methods described below (Patwardhan et al., 2001).

Volumetric image processing. The semiautomated image-processing procedure was conducted with the program BrainImage v5.x (Reiss, 2003) running on an Apple Macintosh G3 or G4 computer. Data processing steps included removal of nonbrain tissues from the images, correction of equipment related image artifacts, separation (segmentation) of tissue components (gray, white, and CSF), normalization of image position, and parcellation of the cerebral cortex into lobe and subcortical regions on the basis of a stereotaxic atlas template (Talairach, 1988). This procedure, described and validated in previous reports (Reiss et al., 1998; Kates et al., 1999), results in volume measurements for gray, white, and CSF total cerebrum, the four cerebral lobes, brainstem, and cerebellum. Although scans were acquired from three different scanners for this project, we have shown previously that our image-processing pipeline is robust and provides consistent results across scanners when similar pulse sequences are used (Patwardhan et al., 2001).

After positional normalization of the brain volume to be parallel to the plane defined by the anterior commissure (AC) and posterior commissure (PC), an additional stereotaxic atlas-based parcellation was used to specify further the prefrontal cortex and subdivide this region into dorsal, superior middle, inferior middle, and orbital components (Fig. 1). For the present analysis, the prefrontal cortex was defined as all frontal gray matter lying anterior to a coronal plane intersecting the most anterior point of the genu of the corpus callosum. In a similar manner, the cingulate gyrus was subdivided into four components corresponding to ventral-anterior, dorsal-anterior, middle, and posterior segments (Fig. 1).

Volumes of additional (i.e., not atlas based) regions of interest (ROIs) were obtained from delineation of specific brain structures by trained research staff who followed a detailed on-line protocol. These ROIs corresponded to brain regions that were designated on an a priori basis to be associated with either the visual-spatial or emotion-face processing networks (see below). Inter-rater reliabilities for all volumes described in this study were $\geq 0.90$ as determined by the intraclass correlation coefficient.

Voxel-based morphometry. Image preprocessing was performed using BrainImage (Stanford Psychiatry Neuroimaging Laboratory, http://spnl.stanford.edu/tools/tools.htm) and SPM99 software (Wellcome Department of Imaging Neuroscience, University College London, London, UK; http://www.fil.ion.ucl.ac.uk/spm/). Skull and other nonbrain tissues were stripped from the images using BrainImage. This step improved image normalization to the template and prevented normalization artifact that can occur because of individual variability in skull shape. SPM99 was used to normalize, segment, and smooth the images. The skullstripped images were normalized to the Montreal Neurological Institute template using a 12 parameter linear affine transformation. The normalized $1 \mathrm{~mm}$ isotropic images were then segmented to create images that represent gray, white, and CSF. Finally, the gray and white matter images were smoothed to $8 \mathrm{~mm}$ full width half maximum to produce normally distributed data. A Gaussian kernel of $8 \mathrm{~mm}$ was used to smooth the data rather than a $12 \mathrm{~mm}$ kernel (Salmond et al., 2002), so that subcortical as well as cortical regions could be compared. There were no predictions about group differences in white matter or CSF and the representative images were not analyzed.

Cognitive assessment. WS participants under 17 years of age $(n=3)$ received the Wechsler Intelligence Scale for Children, Revised Edition (WISC-R), whereas those 17 and older were administered the Wechsler Adult Intelligence Scale, Revised Edition (WAIS-R). The WAIS-R and WISC-R were used, despite the fact that both measures have been revised, 
to maintain backward compatibility with our broader studies of WS cognition that have been ongoing since 1981 .

Data analyses for volumetric variables. Because aspects of visual-spatial information processing occurs within regions of the parietal, temporal, and occipital cortices (Logothetis, 1999), the gray matter volumes of these three lobes along with thalamic gray volume were grouped together for an initial multivariate analysis of visual system anatomy. A similar analysis of gray matter volumes for brain regions comprising the emotion- and face-processing systems included the orbital prefrontal cortex, amygdala, hippocampus, superior temporal gyrus, and four subdivisions of the cingulate gyrus.

All variables were examined for normality to conform to the assumptions of the parametric statistical analyses used. Two initial multivariate analyses of covariance (MANCOVA) were conducted corresponding to the visual and emotion-face-processing systems, respectively, followed by confirmatory univariate ANOVA (or ANCOVA) to evaluate group differences in volumes if the omnibus multivariate $F$ test showed $p \leq$ 0.01 . To minimize type II errors, a two-sided $p$ value of 0.01 also was selected to determine the main effect of group in the confirmatory analyses. Age was used as a standard covariate in all analyses, because many neurodevelopmental processes, such as myelination and synapse elimination, are quite dynamic throughout the age range of the participants (Huttenlocher and Dabholkar, 1997; Paus et al., 2001). Gender also was used as a covariate in these analyses, and models initially included an interaction term for group and gender combined. A more liberal $p$ value of $\leq 0.10$ was used to decide whether the interaction term of group-bygender should be included in the model, because it is well known that statistical power to detect interactions is lower than for main effects.

Investigation of brain asymmetry used repeated measures ANOVA, which took diagnostic category as a between-participant factor and side (left vs right) as a within-participant factor. The interaction effect (group times side) was used to determine group differences in asymmetry. These analyses were used only for regions in which significant between-group differences were demonstrated with univariate confirmatory analyses as described above. SPSS v.11 (SPSS, Chicago, IL) was used for all volumetric analyses, and all $p$ values in this report are of the two-sided type.

Data analysis for voxel-based morphometry. For SPM99-based analyses, voxel-by-voxel unpaired $t$ tests were used to compare signal intensities between groups. To minimize type II error, significant voxels were defined as those exceeding a multiple comparison corrected $p<0.05$ threshold.

\section{Results}

Participant characteristics

A total of 117 scans was acquired, 67 from participants with WS and 50 from healthy controls. Eighty-five scans (45 WS, 40 controls) were considered to be of sufficient quality for additional analysis, whereas 32 (22 WS, 10 control) were judged to be suboptimal because of movement artifact $(n=5)$, the presence of a large cerebellar cyst $(n=1)$, or a scanner-related problem of sufficient magnitude to interfere with image processing (e.g., signal drop out, flow artifact, low dynamic range; $n=26)$. There was no statistically significant difference in the proportion of WS (22 of 67) versus control (10 of 50) scans judged to be unusable. Within the WS group, there was no statistically significant difference in mean full-scale IQ (FSIQ) between participants with usable $(68.7 \pm 9.4)$ and unusable $(64.1 \pm 10.6)$ scans. Two male participants in the WS group were later found to have atypical (smaller) deletions and were removed from the group analyses presented here. Thus, the final groups were comprised of 43 participants with WS (mean age, $28.9 \pm 9.2$ years; range, $12-50$ years; 24 females) and 40 controls (27.5 \pm 7.4 years; range, $18-49$ years; 24 females). The FSIQ scores of 41 WS participants ranged from 46 to 83 with a mean $( \pm S D)$ of $68 \pm 9$. Two participants with WS were not administered the Wechsler Intelligence scales but did exhibit similar levels of cognitive functioning on other measures.
Table 1. Total and regional brain volumes (calculated in cubic centimeters) in WS and control groups

\begin{tabular}{|c|c|c|c|c|}
\hline & WS & & Controls & \\
\hline & Mean & SD & Mean & SD \\
\hline TBV & 1210.5 & 100.5 & 1367.6 & 139.7 \\
\hline Cerebrum & 540.8 & 47.3 & 591.1 & 67.5 \\
\hline Frontal lobe & 196.9 & 19.9 & 210.0 & 23.3 \\
\hline Temporal lobe & 116.3 & 11.5 & 125.3 & 15.3 \\
\hline Parietal lobe & 129.0 & 14.0 & 139.8 & 16.9 \\
\hline Occipital lobe & 60.2 & 8.6 & 73.6 & 13.8 \\
\hline Dorsal prefrontal & 8.3 & 3.1 & 10.8 & 3.4 \\
\hline Middle superior prefrontal & 21.6 & 3.6 & 24.7 & 3.5 \\
\hline Middle inferior prefrontal & 26.2 & 3.5 & 27.6 & 3.6 \\
\hline Orbital prefrontal & 24.2 & 4.9 & 18.2 & 4.6 \\
\hline Superior temporal gyrus & 22.3 & 2.5 & 22.3 & 2.5 \\
\hline Thalamus & 6.9 & 0.8 & 8.3 & 1.1 \\
\hline Amygdala & 3.4 & 0.5 & 3.1 & 0.6 \\
\hline Hippocampus & 5.8 & 0.9 & 5.8 & 0.9 \\
\hline Ventral anterior cingulate & 5.6 & 1.7 & 5.7 & 1.7 \\
\hline Dorsal anterior cingulate & 4.7 & 1.1 & 4.3 & 0.7 \\
\hline Middle cingulate & 3.5 & 0.8 & 3.8 & 0.5 \\
\hline Posterior cingulate & 12.2 & 2.7 & 14.0 & 3.8 \\
\hline
\end{tabular}

Total brain volume is the sum of gray matter, white matter, and CSF. All other values represent gray matter volumes only.

\section{Overall brain volumes}

Total brain volume (TBV) (gray plus white plus CSF) was significantly reduced $(\sim 11.5 \%)$ in WS compared with controls $(F=60.1 ; p<0.0001)$ (Table 1$)$. A multivariate ANOVA incorporating cerebral, cerebellar, and brainstem tissue (i.e., gray plus white) volumes as dependent variables also was significant $(F=31.4 ; p<0.0001)$. Individual follow-up ANOVAs showed cerebral $(p<0.0001)$ and brainstem $(p<0.0001)$ volumes to be significantly reduced in WS but not cerebellar volume $(p=0.15)$.

\section{Visual system}

A MANCOVA was used to test the hypothesis that participants with WS would show reduced occipital cortex and thalamic gray matter volumes compared with controls, even when the effect of overall reduction in total cerebral cortical volume in WS was taken into account statistically. Therefore, this analysis used total cerebral gray matter volume as an additional covariate, whereas the four individual lobe gray matter volumes and thalamic gray volume were used as the dependent variables. Results showed a significant main effect of group (Wilk's $F=6.1 ; p<0.0001$ ) indicating the presence of differing profiles of cortical lobe and thalamic volumes between the groups. The group by gender interaction term was not included in the final model. Examination of the estimated marginal means after statistically adjusting for the effects of the covariates and group differences in overall cerebral cortical gray volume, only the occipital cortex $(p=0.003)$ and thalamus $(p<0.0001)$ were smaller in WS (Figs. 2, 3).

\section{Emotion-face processing}

A MANCOVA was used to investigate the hypothesis that the WS group would show increased volume (relative to overall cerebral size) in regions involved in emotion and face processing. The model used the gray matter volumes of the amygdala, hippocampus, superior temporal gyrus, four subdivisions of the prefrontal cortex, and four subdivisions of the cingulate gyrus as dependent variables and cerebral gray matter volume as an additional covariate. Results showed a significant main effect of group (Wilk's $F=8.2 ; p<0.0001$ ), indicating the presence of differing profiles of regional volumes between the groups. The group by gender 

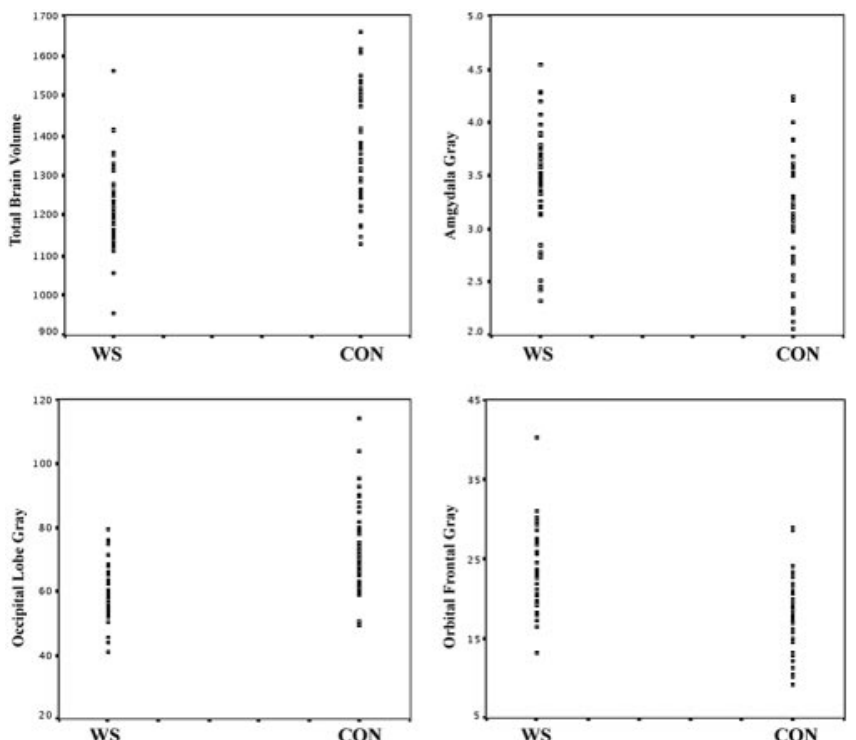

Figure 2. Distribution of brain volumes (calculated in cubic centimeters) in WS and control (Con) groups. Total brain and occipital gray volumes are significantly reduced in WS, whereas amygdala and ventral prefrontal gray volumes are significantly increased compared with controls.
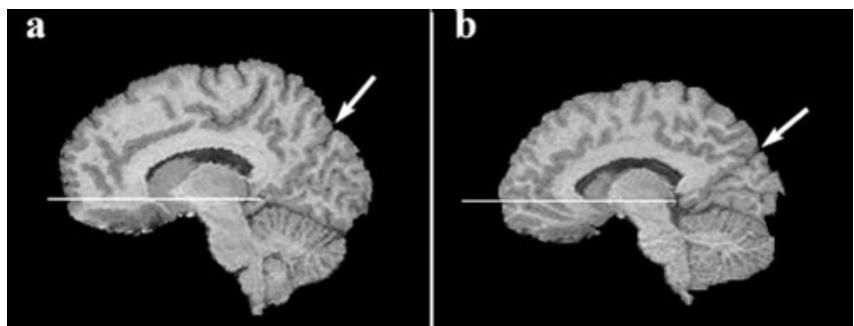

Figure 3. $a, b$, Representative parasagittal images (at the same anatomic location) from a young adult control participant $(a$ ) and an age- and gender-matched participant with Williams syndrome $(b)$. Horizontal line on each image shows the location of the plane defined by the AC and PC. Arrows point to the location of parietal-occipital fissure. Note the relatively greater amount of ventral prefrontal tissue inferior to the $\mathrm{AC}-\mathrm{PC}$ plane and the disproportionately small occipital lobe (posterior and inferior to the parietal-occipital fissure) in the participant with Williams syndrome.

interaction term was not included in the final model. Examination of the estimated marginal means revealed that, after statistically adjusting for overall cerebral gray volume, amygdala ( $p \leq$ $0.001)$, superior temporal gyrus $(p \leq 0.001)$, orbital prefrontal $(p<0.0001)$, and dorsal anterior cingulate $(p \leq 0.005)$, gray volumes were larger in WS participants compared with controls. The scale of larger amygdala and orbital prefrontal volumes in WS was particularly striking; absolute volumes were $\sim 10$ and $30 \%$ larger than controls, respectively, in the context of an overall $11 \%$ reduction in total brain volume (Table 1; Figs. 2, 3 ).

\section{Asymmetry analyses}

Regions shown to differentiate groups in the volumetric analyses described above were evaluated further in analyses evaluating brain asymmetry. The group times side interaction was significant for occipital lobe $(p=0.007)$ gray matter only. This asymmetry difference appeared to be attributable to greater volume reduction in the right occipital cortex in WS leading to a slight left $>$ right volume profile compared with the right $>$ left pattern observed in controls.

Voxel-based morphometry

Table 2 lists the regions and coordinates for clusters where control participants exhibited significantly greater gray matter den- sity than WS participants. Table 2 and Figure $4 a$ demonstrate that these areas included a large cluster focused in bilateral parahippocampal gyri and extending across the midbrain tectum, left superior parietal-occipital cortex, and left insular cortex.

Table 2 also lists the regions and coordinates for clusters where WS participants exhibited significantly greater gray matter density than control participants. The table and Figure $4 b$ demonstrate that these areas included the bilateral orbital frontal gyri, bilateral insula, bilateral fusiform gyrus, the ventral and dorsal anterior cingulate gyrus, medial prefrontal cortex, and cerebellar vermis, including the anterior vermis (lobules I-V), neocerebellar lobules (VI, VII), and the nodulus.

\section{Discussion}

In the context of $11 \%$ reduction in overall cerebral volume compared with controls, regions known to play a role in visual-spatial processing (Logothetis, 1999; Zeki, 2001), the occipital cortex and thalamus, were reduced even further in volume in the WS group. Also, consistent with a hypothesis of visual system abnormalities in WS, reduced gray matter density in the WS group was observed in bilateral parahippocampal gyri, extending across the midbrain tectum (superior and inferior colliculi) and in superior parietal and occipital regions known to be important components of the primate visual system (BA 7 and 19).

As subcortical extrastriate components of the visualperceptual system, the thalamus (pulvinar nucleus) and superior colliculus play important roles in visual-spatial attention and direction of eye movements to "unseen" or masked objects in space (Corbetta et al., 1991; Kustov and Robinson, 1996; Buchel et al., 1998). As suggested in studies of nonhuman primates (Lawler and Cowey, 1986), the reduced volume and gray matter density within regions comprising this secondary visual pathway may contribute to WS deficits in stereopsis and visual acuity (Atkinson et al., 2001) as well as an unusually "intense" gaze when interacting with others (Mervis et al., 2003). Similarly, the parahippocampal region is an essential component of the neural system underlying visual-spatial processing and memory (Aguirre et al., 1998; Epstein and Kanwisher, 1998; Ranganath and D'Esposito, 2001; Davachi et al., 2003).

These findings suggest that both cortical and subcorticalextrastriate visual pathways are adversely affected in WS. In accordance with these findings, a postmortem histological study from Galaburda et al. (2002) showed significantly smaller and more densely packed cells in the peripheral visual cortex of WS brains. Together, histological and gross anatomical abnormalities of the visual system could explain the reduced functional activation within primary and secondary visual cortices during face and gaze processing in WS (Mobbs et al., 2004).

Imaging studies have greatly increased our knowledge of the functional neuroanatomy of emotion (LeDoux, 2000; Phan et al., 2002; Winston et al., 2003), particularly with respect to emotional face processing. There is considerable overlap between brain regions involved in processing of faces and the perception and regulation of emotions including the amygdala, inferior occipitotemporal cortex, cingulate, and prefrontal cortex (Iidaka et al., 2001; Phan et al., 2002; Yamasaki et al., 2002). For this reason and the fact that individuals with WS show heightened or exaggerated behavior in both areas of neurocognitive function, we used an approach that interrogated regions involved in both face and emotion processing.

In support of our second hypothesis, volumetric and VBM analyses showed WS participants to have disproportionately large volumes and increased gray matter density in areas known to be important components of emotion and face processing, 
Table 2. Voxel coordinates in Talairach space, cluster size, and associated Z scores corresponding to brain regions in which significant differences in gray matter density were detected between participants with WS and controls

\begin{tabular}{|c|c|c|c|}
\hline & Cluster size & Zscore & Peak coordinate \\
\hline \multicolumn{4}{|l|}{ Controls $>$ WS } \\
\hline \multicolumn{4}{|l|}{ Bilateral parahippocampal gyrus extending across } \\
\hline midbrain tectum (BA 35) & 2469 & 6.92 & $-12-32-8$ \\
\hline Left superior cerebellar intermediate zone & 636 & 6.88 & $-8-42-22$ \\
\hline Left superior parietal gyrus (BA 7) & 1130 & 6.76 & $-28-66 \quad 36$ \\
\hline Left insula/inferior frontal gyrus (BA 47) & 1096 & 6.66 & $\begin{array}{lll}-30 & 30 & -2\end{array}$ \\
\hline \multicolumn{4}{|l|}{ Left marginal segment of the cingulate/paracen- } \\
\hline tral lobule (BA 7) & 239 & 6.44 & $-16-38 \quad 52$ \\
\hline Left middle occipital gyrus (BA 37) & 199 & 5.92 & $-40-58 \quad 12$ \\
\hline Right superior cerebellar intermediate zone & 538 & 5.89 & $10-42-22$ \\
\hline Left caudate, body & 202 & 5.84 & $-18-14 \quad 26$ \\
\hline Right medial precentral and postcentral gyri (BA 4) & 60 & 5.84 & $18-26 \quad 58$ \\
\hline Septal nuclei & 78 & 5.83 & 22 \\
\hline Left cuneus (BA 19) & 284 & 5.83 & $-6-88 \quad 40$ \\
\hline Right superior parietal gyrus (BA 7) & 119 & 5.68 & $26-62 \quad 36$ \\
\hline Left insula & 22 & 5.63 & $\begin{array}{lll}-36 & -2 & -4\end{array}$ \\
\hline \multicolumn{4}{|l|}{ WS $>$ Controls } \\
\hline Left nodulus & 5754 & $>8$ & $-4-46-34$ \\
\hline Right orbital frontal gyrus (BA 11) & 4859 & $>8$ & $30 \quad 28-20$ \\
\hline Left insula (BA 41) & 1839 & 7.51 & $-30-18 \quad 16$ \\
\hline Left orbital frontal gyrus (BA 11) & 4430 & 7.47 & $-22 \quad 40-18$ \\
\hline Vermis (lobule IV) & 1557 & 7.38 & $1-50$ \\
\hline Left retrosplenial/cingulate gyrus (BA 30) & 986 & 7.21 & $-16-42 \quad 10$ \\
\hline Right cerebellar hemisphere & 1400 & 7.14 & $40-40-32$ \\
\hline Left cingulate gyrus (BA 32) & 2180 & 7.12 & $-4 \quad 26-12$ \\
\hline Left lateral occipital gyrus (BA 18) & 3453 & 7.12 & $-52-74 \quad 0$ \\
\hline Right lateral occipital gyrus (BA 18) & 3040 & 6.85 & $46-88-4$ \\
\hline Left fusiform gyrus (BA 18) & 1218 & 6.76 & $-34-70-8$ \\
\hline Left angular gyrus (BA 39) & 763 & 6.68 & $-42-60 \quad 34$ \\
\hline Left medial frontal gyrus (BA 10) & 372 & 6.58 & $\begin{array}{lll}-6 & 62 & -4\end{array}$ \\
\hline Left cerebellar hemisphere & 866 & 6.49 & $-42-42-30$ \\
\hline Left superior frontal gyrus (BA 10) & 273 & 6.46 & $\begin{array}{lll}-6 & 58 & 10\end{array}$ \\
\hline Left fusiform gyrus (BA 47) & 320 & 6.41 & $-34-46-12$ \\
\hline Vermis (lobule VI) & 317 & 6.33 & $-4-66-10$ \\
\hline Left inferior cerebellar hemisphere & 912 & 6.24 & $-20-68-36$ \\
\hline Left middle temporal gyrus (BA 21) & 119 & 6.20 & $-64-20-10$ \\
\hline Left postcentral gyrus (BA 2) & 294 & 6.06 & $-52-22 \quad 26$ \\
\hline Right postcentral gyrus (BA 2) & 189 & 6.02 & $58-22 \quad 26$ \\
\hline Right fusiform gyrus (BA 37) & 147 & 6.01 & $36-42-16$ \\
\hline Left postcentral gyrus (BA 7) & 112 & 5.93 & $-4-40 \quad 64$ \\
\hline Left superior frontal gyrus (BA 9) & 97 & 5.90 & $\begin{array}{lll}-8 & 52 & 38\end{array}$ \\
\hline Right cingulate gyrus (BA 24) & 323 & 5.74 & $\begin{array}{lll}4 & -8 & 34\end{array}$ \\
\hline Right cingulate gyrus (BA 32) & 63 & 5.60 & $\begin{array}{lll}8 & 44 & 6\end{array}$ \\
\hline Left precuneus (BA 31) & 59 & 5.49 & $-22-68 \quad 26$ \\
\hline
\end{tabular}

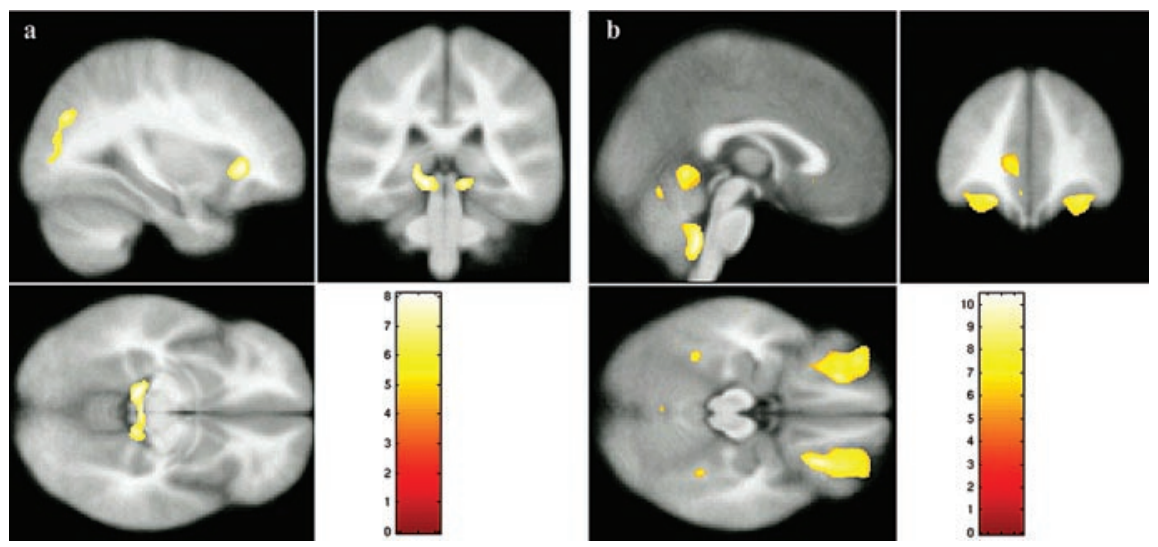

Figure 4. Results of voxel-based morphometry analyses. $a$, Regions where controls showed greater gray matter density compared with WS. $b$, Regions where WS participants showed greater gray matter density compared with controls. namely the amygdala, anterior cingulate cortex, orbital and medial prefrontal cortices, superior temporal gyrus, bilateral fusiform gyri, and insular cortex (Morris et al., 1998; Price, 1999; Bush et al., 2000; Yamasaki et al., 2002).

Grossly increased volumes and increased gray matter densities of the orbital prefrontal cortex, medial prefrontal cortex, and amygdala are of particular interest for understanding the pathogenesis of emotional and behavioral abnormalities in WS. These regions contribute to processing emotion-related and social stimuli and the regulation of appropriate behavioral and autonomic responses to these stimuli (Morris et al., 1998; Price, 1999; Bush et al., 2000; Yamasaki et al., 2002). For example, the amygdala is activated by a broad array of emotions and has important functions in social-affiliative behavior, including facial emotion processing (Davis and Whalen, 2001; Adolphs, 2003).

The amygdala is anatomically linked to orbital prefrontal cortex through robust bidirectional pathways. These pathways are hypothesized to link sensory processing and cognition to emotion (Price, 1999; Ghashghaei and Barbas, 2002). Moreover, the amygdala receives extensive projections from medial prefrontal cortex; this system is thought to contribute to emotional expression, including autonomic nervous system reactivity and endocrine function (Price, 1999; Ghashghaei and Barbas, 2002). To this end, significantly increased gray matter density in bilateral insular cortex in WS is of interest, because this region has been implicated in the modulation of autonomic nervous system output as well (Price, 1999). Collectively, aberrant (augmented) gray matter in orbital and medial prefrontal cortices, amygdale, and insula is in accord with the WSassociated neurobehavioral phenotype that includes hyperarousal, anxiety, altered or enhanced social impulses, enhanced emotional reactivity, and relatively strong face recognition skills. Interestingly, enlargement of the amygdala and orbitofrontal cortex also was reported recently in Turner syndrome (Good et al., 2003), a neurogenetic disorder associated with impaired social cognition and significant visual-spatial deficits.

Increased volume and gray matter density were observed in the anterior cingulate of participants with WS. However, volume increases were limited to the dorsal anterior (cognitive) segment, whereas increased density occurred within the ventral anterior (emotion-related) region (Fig. 4b) (Bush et al., 2000). The lack of 
spatially coincident findings in the cingulate could be associated with methodological differences between the two image analysis techniques. Alternatively, real neuroanatomical differences within disparate regions of the cingulate may have been highlighted by each of the two complementary image analysis strategies. For example, a greater incidence of a paracingulate (i.e., fissurized or split) sulcal pattern (Yucel et al., 2001) in WS could, in part, account for increased volume within the dorsal anterior cingulate. Conversely, greater gray matter density within the ventral anterior cingulate may be a long-term outcome of attentional focus on emotional stimuli leading to increased neuropil in this region.

Although the volume of the fusiform region was not measured in this study, increased gray density was observed in the WS group within bilateral fusiform gyri. Although some debate remains as to the precise role of the fusiform in human perception, the majority of studies indicate a major role in face recognition and processing (Clark et al., 1996; Kanwisher et al., 1997; Tarr and Gauthier, 2000). The fusiform also appears to be sensitive to emotional valence and shows increased activation when visual stimuli have emotional content or when a cognitive task requires attribution of emotion from facial expressions (Critchley et al., 2000; Geday et al., 2003). Therefore, increased gray matter density in the fusiform is consistent with WS-heightened responses to faces and emotional stimuli in general.

\section{Limitations}

VBM analyses identified a number of predicted group differences but also several unexpected regions where WS exhibited greater gray matter density compared with control participants. Some of these results are consistent with previously reported anatomic variations of the WS brain such as increased size of the cerebellar vermis and hemispheres (Wang et al., 1992b; Reiss et al., 2000; Schmitt et al., 2001b). Others such as increased gray matter density in the lateral occipital lobe may stem from localized differences in WS brain shape (Schmitt et al., 2001a). In our study, we chose to normalize images for the VBM analyses with a linear transformation out of concern that use of a nonlinear transformation would complicate further the interpretation of the results. We are currently working to characterize and quantify shape differences in the WS brain using automated methods (Thompson et al., 2001) to characterize more clearly brain regions that exhibit shape differences because of abnormal brain development.

The WS and healthy control difference in IQ is also a study limitation. IQ might be associated with anatomical differences between the groups such as reduced total brain volume in WS. Although the particular topography of neuroanatomical variation we observed in WS has not been reported yet in other neurogenetic or neurodevelopmental disorders, the specificity of individual anatomical variation in WS (such as amygdalar enlargement) also should be elucidated through comparisons to specific and nonspecific groups with comparable IQ.

\section{Conclusions and synthesis}

Together, volumetric and gray matter density reductions within several cortical and subcortical regions comprising the visual system in WS point to a neuroanatomical correlate for the visualspatial deficits associated with this disorder. Likewise, increased volume or gray matter density within brain regions known to participate in processing of emotion and face stimuli may underlie the islands of preserved skills or inappropriately heightened and exaggerated behaviors relating to these cognitive areas. Although it is relatively straightforward to consider how particular brain regions might exhibit reduced volume or gray matter density in association with an adverse genetic or other biological factor, a more complex issue is how augmented brain areas relate to hemizygosity for one or more genes in the WS critical region of chromosome 7.

The specific answer to how and why the WS brain develops in the manner illustrated in this study remains to be answered by integrated scientific inquiry at the level of the gene, cell, brain, and behavior. Within the realm of imaging specifically, longitudinal studies involving very young children with WS, and functional as well as structural imaging, are needed to elucidate the enigma of brain structure-function relationships in this disorder. We predict that these new methods will not only bring a significant expansion in our understanding of the pathophysiology of WS but also will greatly enrich our knowledge of human genebrain-behavior mechanisms in general.

\section{References}

Adolphs R (2003) Is the human amygdala specialized for processing social information? Ann NY Acad Sci 985:326-340.

Aguirre GK, Zarahn E, D’Esposito M (1998) Neural components of topographical representation. Proc Natl Acad Sci USA 95:839-846.

Atkinson J, King J, Braddick O, Nokes L, Anker S, Braddick F (1997) A specific deficit of dorsal stream function in Williams' syndrome. NeuroReport 8:1919-1922.

Atkinson J, Anker S, Braddick O, Nokes L, Mason A, Braddick F (2001) Visual and visuospatial development in young children with Williams syndrome. Dev Med Child Neurol 43:330-337.

Bellugi U, Lichtenberger L, Mills D, Galaburda A, Korenberg JR (1999) Bridging cognition, the brain and molecular genetics: evidence from Williams syndrome. Trends Neurosci 22:197-207.

Bellugi U, Lichtenberger L, Jones W, Lai Z, St. George M (2000) I. The neurocognitive profile of Williams Syndrome: a complex pattern of strengths and weaknesses. J Cogn Neurosci 12[Suppl 1]:7-29.

Buchel C, Josephs O, Rees G, Turner R, Frith CD, Friston KJ (1998) The functional anatomy of attention to visual motion. A functional MRI study. Brain 121:1281-1294.

Bush G, Luu P, Posner MI (2000) Cognitive and emotional influences in anterior cingulate cortex. Trends Cogn Sci 4:215-222.

Clark VP, Keil K, Maisog JM, Courtney S, Ungerleider LG, Haxby JV (1996) Functional magnetic resonance imaging of human visual cortex during face matching: a comparison with positron emission tomography. NeuroImage 4:1-15.

Corbetta M, Miezin FM, Dobmeyer S, Shulman GL, Petersen SE (1991) Selective and divided attention during visual discriminations of shape, color, and speed: functional anatomy by positron emission tomography. J Neurosci 11:2383-2402.

Critchley H, Daly E, Phillips M, Brammer M, Bullmore E, Williams S, Van Amelsvoort T, Robertson D, David A, Murphy D (2000) Explicit and implicit neural mechanisms for processing of social information from facial expressions: a functional magnetic resonance imaging study. Hum Brain Mapp 9:93-105.

Davachi L, Mitchell JP, Wagner AD (2003) Multiple routes to memory: distinct medial temporal lobe processes build item and source memories. Proc Natl Acad Sci USA 100:2157-2162.

Davies M, Udwin O, Howlin P (1998) Adults with Williams syndrome. Preliminary study of social, emotional and behavioural difficulties. Br J Psychiatry 172:273-276.

Davis M, Whalen PJ (2001) The amygdala: vigilance and emotion. Mol Psychiatry 6:13-34.

Dykens EM (2003) Anxiety, fears, and phobias in persons with Williams syndrome. Dev Neuropsychol 23:291-316.

Einfeld SL, Tonge BJ, Florio T (1997) Behavioral and emotional disturbance in individuals with Williams syndrome. Am J Ment Retard 102:45-53.

Epstein R, Kanwisher N (1998) A cortical representation of the local visual environment. Nature 392:598-601.

Galaburda AM, Holinger DP, Bellugi U, Sherman GF (2002) Williams syndrome: neuronal size and neuronal-packing density in primary visual cortex. Arch Neurol 59:1461-1467.

Geday J, Gjedde A, Boldsen AS, Kupers R (2003) Emotional valence modulates activity in the posterior fusiform gyrus and inferior medial prefrontal cortex in social perception. NeuroImage 18:675-684. 
Ghashghaei HT, Barbas H (2002) Pathways for emotion: interactions of prefrontal and anterior temporal pathways in the amygdala of the rhesus monkey. Neuroscience 115:1261-1279.

Good CD, Johnsrude IS, Ashburner J, Henson RNA, Friston KJ, Frackowiak RSJ (2001) A voxel-based morphometric study of ageing in 465 normal adult human brains. NeuroImage 14:21-36.

Good CD, Lawrence K, Thomas NS, Price CJ, Ashburner J, Friston KJ, Frackowiak RS, Oreland L, Skuse DH (2003) Dosage-sensitive X-linked locus influences the development of amygdala and orbitofrontal cortex, and fear recognition in humans. Brain 126:2431-2446.

Gosch A, Pankau R (1994) Social-emotional and behavioral adjustment in children with Williams-Beuren syndrome. Am J Med Genet 53:335-339.

Hopyan T, Dennis M, Weksberg R, Cytrynbaum C (2001) Music skills and the expressive interpretation of music in children with Williams-Beuren syndrome: pitch, rhythm, melodic imagery, phrasing, and musical affect. Neuropsychol Dev Cogn Sect C Child Neuropsychol 7:42-53.

Huttenlocher PR, Dabholkar AS (1997) Regional differences in synaptogenesis in human cerebral cortex. J Comp Neurol 387:167-178.

Iidaka T, Omori M, Murata T, Kosaka H, Yonekura Y, Okada T, Sadato N (2001) Neural interaction of the amygdala with the prefrontal and temporal cortices in the processing of facial expressions as revealed by fMRI. J Cogn Neurosci 13:1035-1047.

Jones W, Bellugi U, Lai Z, Chiles M, Reilly J, Lincoln A, Adolphs R (2000) II. Hypersociability in Williams Syndrome. J Cogn Neurosci 12[Suppl 1]:30-46.

Kanwisher N, McDermott J, Chun MM (1997) The fusiform face area: a module in human extrastriate cortex specialized for face perception. J Neurosci 17:4302-4311.

Kates WR, Warsofsky IS, Patwardhan A, Abrams MT, Liu AM, Naidu S, Kaufmann WE, Reiss AL (1999) Automated Talairach atlas-based parcellation and measurement of cerebral lobes in children. Psychiatry Res 91:11-30.

Kustov AA, Robinson DL (1996) Shared neural control of attentional shifts and eye movements. Nature 384:74-77.

Lawler KA, Cowey A (1986) The effects of pretectal and superior collicular lesions on binocular vision. Exp Brain Res 63:402-408.

LeDoux JE (2000) Emotion circuits in the brain. Annu Rev Neurosci 23:155-184.

Levitin DJ, Menon V, Schmitt JE, Eliez S, White CD, Glover GH, Kadis J, Korenberg JR, Bellugi U, Reiss AL (2003) Neural correlates of auditory perception in Williams syndrome: an fMRI study. NeuroImage 18:74-82.

Logothetis NK (1999) Vision: a window on consciousness. Sci Am 281:69-75.

Losh M, Bellugi U, Reilly J, Anderson D (2000) Narrative as a social engagement tool: the excessive use of evaluation in narratives from children with Williams syndrome. Narrative Inquiry 10:1-26.

Mervis CB, Morris CA, Klein-Tasman BP, Bertrand J, Kwitny S, Appelbaum LG, Rice CE (2003) Attentional characteristics of infants and toddlers with Williams syndrome during triadic interactions. Dev Neuropsychol 23:243-268.

Mobbs D, Garrett AS, Menon V, Rose FE, Bellugi U, Reiss AL (2004) Anomalous brain activation during face and gaze processing in Williams syndrome. Neurology 62:2070-2076.

Morris JS, Friston KJ, Buchel C, Frith CD, Young AW, Calder AJ, Dolan RJ (1998) A neuromodulatory role for the human amygdala in processing emotional facial expressions. Brain 121:47-57.

Patwardhan AJ, Eliez S, Warsofsky IS, Glover GH, White CD, Giedd JN, Peterson BS, Rojas DC, Reiss AL (2001) Effects of image orientation on the comparability of pediatric brain volumes using three-dimensional MR data. J Comput Assist Tomogr 25:452-457.

Paul BM, Stiles J, Passarotti A, Bavar N, Bellugi U (2002) Face and place processing in Williams syndrome: evidence for a dorsal-ventral dissociation. NeuroReport 13:1115-1119.

Paus T, Collins DL, Evans AC, Leonard G, Pike B, Zijdenbos A (2001) Maturation of white matter in the human brain: a review of magnetic resonance studies. Brain Res Bull 54:255-266.

Phan KL, Wager T, Taylor SF, Liberzon I (2002) Functional neuroanatomy of emotion: a meta-analysis of emotion activation studies in PET and fMRI. NeuroImage 16:331-348.

Price JL (1999) Prefrontal cortical networks related to visceral function and mood. Ann NY Acad Sci 877:383-396.

Ranganath C, D’Esposito M (2001) Medial temporal lobe activity associated with active maintenance of novel information. Neuron 31:865-873.

Reilly J, Losh M, Bellugi U, Wulfeck B (2004) "Frog where are you?” Narratives in children with specific language impairment, early focal brain injury and Williams syndrome. Brain Lang 88:229-247.

Reiss AL, Hennessey JG, Rubin M, Beach L, Abrams MT, Warsofsky IS, Liu AM, Links JM (1998) Reliability and validity of an algorithm for fuzzy tissue segmentation of MRI. J Comput Assist Tomogr 22:471-479.

Reiss AL, Eliez S, Schmitt JE, Straus E, Lai Z, Jones W, Bellugi U (2000) IV. Neuroanatomy of Williams syndrome: a high-resolution MRI study. J Cogn Neurosci 12[Suppl 1]:65-73.

Salmond CH, Ashburner J, Vargha-Khadem F, Connelly A, Gadian DG, Friston KJ (2002) Distributional assumptions in voxel-based morphometry. NeuroImage 17:1027-1030.

Schmitt JE, Eliez S, Bellugi U, Reiss AL (2001a) Analysis of cerebral shape in Williams syndrome. Arch Neurol 58:283-287.

Schmitt JE, Eliez S, Warsofsky IS, Bellugi U, Reiss AL (2001b) Enlarged cerebellar vermis in Williams syndrome. J Psychiatr Res 35:225-229.

Schmitt JE, Eliez S, Warsofsky IS, Bellugi U, Reiss AL (2001c) Corpus callosum morphology of Williams syndrome: relation to genetics and behavior. Dev Med Child Neurol 43:155-159.

Schmitt JE, Watts K, Eliez S, Bellugi U, Galaburda AM, Reiss AL (2002) Increased gyrification in Williams syndrome: evidence using 3D MRI methods. Dev Med Child Neurol 44:292-295.

Talairach JT (1988) Co-planar stereotaxic atlas of the human brain atlas. New York: Theime.

Tarr MJ, Gauthier I (2000) FFA: a flexible fusiform area for subordinatelevel visual processing automatized by expertise. Nat Neurosci 3:764-769.

Thompson PM, Cannon TD, Narr KL, van Erp T, Poutanen VP, Huttunen M, Lonnqvist J, Standertskjold-Nordenstam CG, Kaprio J, Khaledy M, Dail R, Zoumalan CI, Toga AW (2001) Genetic influences on brain structure. Nat Neurosci 4:1253-1258.

Wang PP, Doherty S, Hesselink JR, Bellugi U (1992a) Callosal morphology concurs with neurobehavioral and neuropathological findings in two neurodevelopmental disorders. Arch Neurol 49:407-411.

Wang PP, Hesselink JR, Jernigan TL, Doherty S, Bellugi U (1992b) Specific neurobehavioral profile of Williams' syndrome is associated with neocerebellar hemispheric preservation. Neurology 42:1999-2002.

Winston JS, O'Doherty J, Dolan RJ (2003) Common and distinct neural responses during direct and incidental processing of multiple facial emotions. NeuroImage 20:84-97.

Yamasaki H, LaBar KS, McCarthy G (2002) Dissociable prefrontal brain systems for attention and emotion. Proc Natl Acad Sci USA 99:11447-11451.

Yucel M, Stuart GW, Maruff P, Velakoulis D, Crowe SF, Savage G, Pantelis C (2001) Hemispheric and gender-related differences in the gross morphology of the anterior cingulate/paracingulate cortex in normal volunteers: an MRI morphometric study. Cereb Cortex 11:17-25.

Zeki S (2001) Localization and globalization in conscious vision. Annu Rev Neurosci 24:57-86. 